\title{
Frequency of symptomatic vertebral body compression fractures requiring intervention following single-fraction stereotactic radiosurgery for spinal metastases
}

\author{
Michael S. Virk, MD, PhD, ${ }^{1,2}$ James E. Han, MD, ${ }^{3}$ Anne S. Reiner, $M P H,{ }^{4}$ Lily A. McLaughlin, BS, ${ }^{1}$ \\ Daniel M. Sciubba, MD, ${ }^{5}$ Eric Lis, MD, ${ }^{6}$ Yoshiya Yamada, MD, ${ }^{3}$ Mark Bilsky, MD, ${ }^{1,2}$ and \\ llya Laufer, MD1,2
}

Departments of ${ }^{1}$ Neurological Surgery, ${ }^{3}$ Radiation Oncology, ${ }^{4}$ Epidemiology and Biostatistics, and ${ }^{6}$ Radiology, Memorial Sloan Kettering Cancer Center; ${ }^{2}$ Department of Neurological Surgery, Weill Cornell Medical College, NewYork-Presbyterian Hospital, New York, New York; and ${ }^{5}$ Department of Neurosurgery, Johns Hopkins University School of Medicine, Baltimore, Maryland

OBJECTIVE The purpose of this study was to determine the rate of symptomatic vertebral body compression fractures (VCFs) requiring kyphoplasty or surgery in patients treated with 24-Gy single-fraction stereotactic radiosurgery (SRS).

METHODS This retrospective analysis included all patients who had been treated with 24-Gy, single-fraction, imageguided intensity-modulated radiation therapy for histologically confirmed solid tumor metastases over an 8-year period (2005-2013) at Memorial Sloan Kettering Cancer Center. Charts and imaging studies were reviewed for post-SRS kyphoplasty or surgery for mechanical instability. A Spinal Instability Neoplastic Score (SINS) was calculated for each patient both at the time of SRS and at the time of intervention for VCF.

RESULTS Three hundred twenty-three patients who had undergone single-fraction SRS between C-1 and L-5 were included in this analysis. The cumulative incidence of VCF 5 years after SRS was $7.2 \%(95 \% \mathrm{Cl} 4.1-10.2)$, whereas that of death following SRS at the same time point was $82.5 \%$ (95\% CI 77.5-87.4). Twenty-six patients with 36 SRS-treated levels progressed to symptomatic VCF requiring treatment with kyphoplasty (6 patients), surgery (10 patients), or both (10 patients). The median time to symptomatic VCF was 13 months. Seven patients developed VCF at 11 levels adjacent to the SRS-treated level. Fractured levels had no evidence of tumor progression. The median SINS changed from 6.5 at SRS (interquartile range [IQR] 4.3-8.8) to 11.5 at stabilization (IQR 9-13). In patients without prior stabilization at the level of SRS, there was an association between the SINS and the time to fracture.

CONCLUSIONS Five years after ablative single-fraction SRS to spinal lesions, the cumulative incidence of symptomatic VCF at the treated level without tumor recurrence was $7.2 \%$. Higher SINSs at the time of SRS correlated with earlier fractures.

https://thejns.org/doi/abs/10.3171/2016.10.FOCUS16359

KEY WORDS stereotactic radiosurgery; vertebral body fracture; complications; kyphoplasty

$\mathrm{T}$ HE spine is the most common site of osseous metastatic cancer and represents a significant source of morbidity in patients with disseminated disease. Spinal radiosurgery has an expanding role in treatment paradigms for metastases, with its safety and efficacy established in numerous publications. Single-fraction stereotactic radiosurgery (SRS) is increasingly used to deliver ablative radiation doses to tumors that show resistance to conventionally fractionated radiation. With the growing implementation of SRS, radiation-associated toxicity must be carefully studied and delineated to optimize the safety of this therapy. Complications of spinal SRS include fatigue, dermatitis, esophagitis, myelopathy, radiculopathy, and vertebral body compression fracture (VCF). The overwhelming majority of toxicities associated with spinal SRS are low grade, with significant neurological or esophageal toxicity being a very rare occurrence. Radiation-associated VCF has been described in several publications and its incidence ranges from 5.7\% to 39\%., ${ }^{3,5,8,15,17,20}$ Most studies have analyzed patients treated with a broad range of ra-

ABBREVIATIONS IG-IMRT = image-guided IMRT; IMRT = intensity-modulated radiation therapy; IQR = interquartile range; KPS = Karnofsky Performance Scale; SINS = Spinal Instability Neoplastic Score; SRS = stereotactic radiosurgery; VCF = vertebral body compression fracture.

SUBMITTED August 26, 2016. ACCEPTED October 11, 2016.

INCLUDE WHEN CITING DOI: 10.3171/2016.10.FOCUS16359. 
diation doses and have examined the risk of radiographic fracture development rather than clinically symptomatic fractures. The purpose of the present study was to identify the rate of symptomatic VCFs that require stabilization with kyphoplasty or surgery in a large population treated with a uniform dose of $24 \mathrm{~Gy}$ in a single fraction. While radiographic fractures may be asymptomatic and may not have clear implications for the clinical course of patients, the determination of clinically significant VCFs may help to guide clinical practice and help patients to make informed treatment decisions.

\section{Methods}

Treatment technique and dosimetry for radiosurgery have been described elsewhere. ${ }^{6}$ In brief, all patients in the current study were treated with image guidance, and since 2005 in-room CT-on-rails or cone-beam imaging has been used for treatment verification. Inverse treatment planning algorithms for intensity-modulated radiation therapy (IMRT) were used for every case, with the optimized isodose line normalized to the $100 \%$ isodose line (prescription dose). Volumetric modulated arc therapy (VMAT) has been the standard beam arrangement since 2014. Before then, 5-7 fixed IMRT beams made up the typical beam arrangement. Table 1 lists the normal tissue constraints and treatment planning dose goals currently in use. Spinal cord contours are typically defined based on a CT myelogram used for simulation. Customized immobilization is used for each case, with patients positioned supine on an alpha cradle with lateral supports.

The institutional review board at Memorial Sloan Kettering Cancer Center approved the current study. Given the retrospective nature of the study, a patient consent waiver was granted by the institution. The retrospective analysis included all patients who had been treated with 24-Gy, single-fraction image-guided (IG)-IMRT for histologically confirmed solid tumor metastases over an 8-year period (2005-2013) at Memorial Sloan Kettering Cancer Center. The primary outcome of analysis was the development of a clinically symptomatic VCF requiring stabilization at or adjacent to the level treated with SRS. Charts and imaging studies were reviewed. Patients who developed local recurrence after SRS were excluded from analysis; therefore, patients requiring surgery for tumor recurrence and patients with a biopsy indicating viable tumor at the time of stabilization were not considered as cases of post-SRS instability. Among the patients who required stabilization, a Spinal Instability Neoplastic Score (SINS) was calculated for each at the time of SRS and at the time of intervention for the VCF. Image-guided IMRT was performed according to standard techniques described in the literature. ${ }^{22}$

Microsoft Excel version 14.4.0 (Microsoft Corp.), IBM SPSS version 22 (IBM Corp.), and R version 3.1.2 (R Foundation) using the 'cmprsk' package were used for statistical analysis and graphics. The association between SINS and time to fracture was tested using linear regression analysis. Competing-risks analysis was used to calculate cumulative incidence and $95 \%$ confidence intervals for VCF and death. The VCF was the event of interest, and
TABLE 1. Dose constraints for single-fraction spine radiosurgery (no prior radiotherapy)

\begin{tabular}{l}
\hline Maximum doses by organ \\
\hline Spinal cord: Dmax $14 \mathrm{~Gy}, \mathrm{D} 0.35 \mathrm{~cm}^{3}<10 \mathrm{~Gy}$ \\
\hline Cauda equina: Dmax $18 \mathrm{~Gy}, \mathrm{D} 5 \mathrm{~cm}^{3} \leq 14 \mathrm{~Gy}$ \\
\hline Esophagus: D2.5 cm $\mathrm{cm}^{3} \leq \mathrm{V} 14 \mathrm{~Gy}$ \\
\hline Brachial plexus: Dmax $14 \mathrm{~Gy}$ \\
\hline Heart: Dmax $22 \mathrm{~Gy}, \mathrm{D} 15 \mathrm{~cm}^{3} \leq 16 \mathrm{~Gy}$ \\
\hline Stomach: Dmax $15 \mathrm{~Gy}, \mathrm{D} 5 \mathrm{~cm}^{3} \leq 12 \mathrm{~Gy}$ \\
\hline Bowel: Dmax $16 \mathrm{~Gy}, \mathrm{D} 5 \mathrm{~cm}^{3} \leq 12 \mathrm{~Gy}$ \\
\hline Kidney: V10 Gy $\leq 33 \%$ of spared kidney vol \\
\hline Rectum: Dmax $\leq 16 \mathrm{~Gy}$ \\
\hline Planning objectives \\
\hline PTV coverage \\
\hline Ideal: V100 $\geq 90 \% \&$ V95 $\geq 95 \%$ \\
\hline Acceptable: V100 $\geq 80 \%$ \& V $95 \geq 90 \%$ \\
\hline PTV inhomogeneity \\
\hline Max point dose: $110 \%-130 \%$ \\
\hline Global Dmax must be w/in PTV \\
\hline High dose spillage \\
\hline Hotspots $\geq 115 \%$ should be w/in PTV \\
\hline Hotspots $\geq 105 \%$ outside of PTV should be limited to $3 \mathrm{~cm}^{3}$ \\
\hline
\end{tabular}

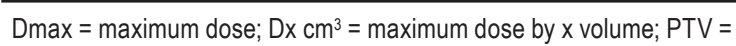
planning target volume; $\mathrm{Vx}=\mathrm{x} \%$ of the planning target volume gets the dose indicated, for example, $90 \%$ or $10 \mathrm{~Gy}$.

death was the competing event. Time to VCF or death was calculated from the date of SRS. Data presented in the text and tables are expressed as medians with interquartile ranges (IQRs 1st-3rd), unless otherwise noted.

\section{Results}

\section{Patient Population}

The study included 323 patients who had undergone SRS at 24 Gy in a single fraction between C-1 and L-5 (Table 2). Median patient age at the time of SRS was 60.7 years (51.6-69.7 years), and overall median follow-up from the date of SRS was 12.6 months (3.7-31.9 months). At the time of our analysis, $259(80.2 \%)$ of the 323 patients were dead, and their median survival was 11 months. The median follow-up among the patients who were alive at the time of our analysis was 34 months. The most common primary tumors included renal cell (66 tumors), prostate (35), sarcoma (32), melanoma (26), non-small cell lung (25), colon (25), breast (19), and thyroid (18). None of the included patients had a history of previous radiation.

Of the 323 patients, 26 experienced a VCF requiring treatment with kyphoplasty (6 patients), surgery (10 patients), or both (10 patients). The cumulative incidence of VCF 5 years after SRS was 7.2\% (95\% CI 4.1-10.2), while that of death following SRS at the same time point was 82.5\% (95\% CI 77.5-87.4; Fig. 1 and Table 3).

A total of 45 VCFs occurred among the 552 treated levels (Table 4). Seven patients had 11 VCFs (2.0\% of 552 levels) that occurred adjacent to the SRS-treated levels. Twenty-eight VCFs were de novo (62\%), while the remainder 
TABLE 2. Demographics of 323 patients who underwent singlefraction SRS for spinal metastasis

\begin{tabular}{lc}
\hline \multicolumn{1}{c}{ Characteristic } & \multicolumn{1}{c}{ Value } \\
\hline No. of SRS-treated vertebral levels & 552 \\
\hline Sex (M/F) & $201 / 121$ \\
\hline Median patient age at SRS in yrs (IQR) & $60.7(51.6-69.7)$ \\
\hline Median time from SRS to VCF in mos (IQR) & $13.2(6.3-28.7)$ \\
\hline Median follow-up from SRS in mos (IQR) & $12.6(3.7-31.9)$ \\
\hline Median survival after SRS in mos (IQR) & $11.1(4.4-23.8)$ \\
\hline Median follow-up for survivors in mos (IQR) & $33.7(7.6-50.4)$ \\
\hline Total patients deceased & $259(80.2 \%)$ \\
\hline Patients undergoing kyphoplasty prior to SRS & $17(5.3 \%)$ \\
\hline Patients undergoing (separation) surgery prior to & $37(11.4 \%)$ \\
\hline SRS & \\
\hline Total recurrences at SRS-treated level & $6.5 \%(21 / 323)$ \\
\hline Surgery for recurrence & $100 \%(21 / 21)$ \\
\hline Progression at level adjacent to SRS-treated level & $1.2 \%(4 / 323)$ \\
\hline Surgery for adjacent-level progression & $100 \%(4 / 4)$ \\
\hline Histology of patients treated w/ SRS & \\
\hline Renal & $66(20 \%)$ \\
\hline Prostate & $35(11 \%)$ \\
\hline Sarcoma & $32(10 \%)$ \\
\hline Melanoma & $26(8 \%)$ \\
\hline Non-small cell lung & $25(8 \%)$ \\
\hline Colon \& rectal & $25(8 \%)$ \\
\hline Breast & $19(6 \%)$ \\
\hline Thyroid & $18(6 \%)$ \\
\hline Chordoma & $11(3 \%)$ \\
\hline Other & $66(20 \%)$ \\
\hline &
\end{tabular}

(38\%) represented progression of existing fractures noted at the time of SRS. There was 1 cervical VCF $(1.7 \%$ of 58 treated levels), 16 thoracic (5.0\% of 322 levels), 27 lumbar (15.2\% of 178 levels), and 1 sacral (adjacent level). Among the patients with VCF, the primary tumors included renal cell carcinoma (7 patients), non-small cell lung carcinoma (4 patients), and prostate cancer, breast cancer, melanoma, and sarcoma (3 patients each), as well as thyroid, paraganglioma, and Hürthle cell adenoma (1 patient each; Table 4). The median time from SRS to symptomatic VCF was 13.2 months (6.3-28.7 months).

In 24 patients, biopsy at the time of stabilization showed no evidence of tumor. The 2 remaining patients had no specimen taken at the time of stabilization, but neither did they have evidence of radiographic or clinical progression. Twenty-one patients with VCF were excluded from analysis because of tumor progression at the SRS-treated levels; these patients were taken to surgery for repeat decompressions. An additional 4 patients were taken to surgery for tumor progression at levels adjacent to the SRS-treated levels. Thirty-seven (11.4\%) of the 323 patients had had separation surgery prior to SRS, and 17 (5.3\%) had had kyphoplasty prior to SRS (Table 2).

Among the 26 patients who sustained a post-SRS VCF, 7 had undergone stabilization (kyphoplasty or surgery) of

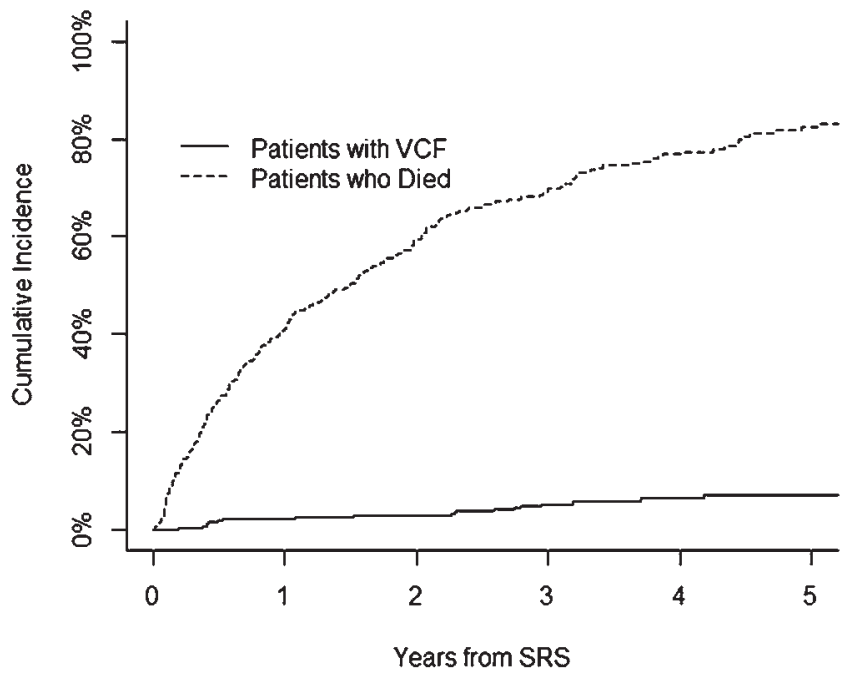

FIG. 1. Cumulative incidence curves for VCF and death following singlefraction SRS 24 Gy. Competing-risks analysis was performed with VCF designated as the event of interest and death as the competing event. The time to VCF or death was calculated from the date of SRS. Cumulative incidence values and $95 \%$ confidence intervals at various time points are listed in Table 3.

the treated level before undergoing SRS and subsequently had fracture progression at the index level. These patients were treated either with salvage kyphoplasty in the postinstrumentation setting or with instrumented stabilization for more extensive fractures. One of the patients underwent repeat kyphoplasty after fracture progression at a vertebral level with a previous kyphoplasty. The remaining 19 patients underwent SRS as an upfront treatment. Among the patients stabilized pre-SRS, 3 had symptomatic compression fractures that were treated with kyphoplasty before undergoing radiation, while the other 4 patients un-

TABLE 3. Cumulative incidence of VCF and death following SRS

\begin{tabular}{cc}
\hline VCF Following SRS & Cumulative Incidence $(95 \% \mathrm{Cl})$ \\
\hline Time from SRS & \\
\hline 3 mos & $0.31(0-0.93)$ \\
\hline 6 mos & $1.9(0.4-3.4)$ \\
\hline 1 yr & $2.2(0.6-3.8)$ \\
\hline 2 yrs & $2.9(1.0-4.7)$ \\
\hline 3 yrs & $5.4(2.8-7.9)$ \\
\hline 4 yrs & $6.6(3.7-9.6)$ \\
\hline 5 yrs & $7.2(4.1-10.2)$ \\
\hline Death following SRS & \\
\hline Time from SRS & $14.7(10.8-18.6)$ \\
\hline 3 mos & $26.7(21.8-31.6)$ \\
\hline 6 mos & $41.2(35.8-46.7)$ \\
\hline 1 yr & $59.3(53.8-64.8)$ \\
\hline 2 yrs & $69.7(64.5-74.9)$ \\
\hline 3 yrs & $76.8(71.8-81.8)$ \\
\hline 4 yrs & $82.5(77.5-87.4)$ \\
\hline 5 yrs &
\end{tabular}


TABLE 4. Characteristics of patients with VCF after SRS

\begin{tabular}{ll}
\hline \multicolumn{1}{c}{ Parameter } & \multicolumn{1}{c}{ Value } \\
\hline Patients w/ VCF after SRS & $8.0 \%(26 / 323)$ \\
\hline Levels w/ VCF after SRS & $8.2 \%(45 / 552)$ \\
\hline Adjacent-level fractures & $2.0 \%(11 / 552)$ \\
\hline Patients w/ VCF treated w/ kyphoplasty alone & 6 \\
\hline Patients w/ VCF treated w/ surgery alone & 10 \\
\hline Patients w/ VCF treated w/ surgery \& kyphoplasty & 10 \\
\hline Histology of patients w/ VCF & \\
\hline Renal cell carcinoma & $7(27 \%)$ \\
\hline Non-small cell lung cancer & $4(15 \%)$ \\
\hline Sarcoma & $3(12 \%)$ \\
\hline Prostate cancer & $3(12 \%)$ \\
\hline Melanoma & $3(12 \%)$ \\
\hline Breast cancer & $3(12 \%)$ \\
\hline Other & $3(12 \%)$ \\
\hline
\end{tabular}

derwent SRS as adjuvant treatment after undergoing spinal decompression and instrumented stabilization, that is, separation surgery. Among the 19 patients who underwent upfront SRS, the median SINS at the time of SRS was 6.5 (4.3-8.8). After they sustained a symptomatic VCF and required stabilization, their median SINS was 11.5 (9-13). Representative kyphoplasty and surgical cases are featured in Figs. 2 and 3. There was a linear correlation between the SINS score at the time of SRS and the time to fracture in these 19 patients $\left(\mathrm{R}^{2}=0.4, \mathrm{p}<0.001\right.$; Fig. 4$)$.

\section{Discussion}

This retrospective analysis of 323 patients with 552 vertebral levels receiving single-fraction (24 Gy/fraction) SRS revealed a 7.2\% cumulative incidence of VCF. Seven patients sustained $11 \mathrm{VCF}$ at levels adjacent to the SRStreated level. Median time from SRS to symptomatic VCF was 13.2 months. A competing-risks analysis was used to examine the cumulative incidence of VCF versus death at multiple time points. Importantly, the symptomatic VCF group presented here includes patients who required procedural intervention but excludes those who underwent surgery for tumor recurrence. Among the patients with tumor recurrence, progression of fracture cannot with certainty be attributed to post-SRS instability since tumor progression contributes to instability. The focus of our analysis was the development of gross mechanical instability attributable to the effect of SRS on treated vertebrae. Thus, the 8\% VCF incidence following single-fraction (24 Gy/fraction) SRS reported in the current study cannot be attributed to disease progression.

Previous publications have reported that $5.7 \%-39 \%$ of vertebral bodies treated with SRS exhibit radiographic occurrence or progression of VCF (Table 5). ${ }^{3,5,8,15,17,20} \mathrm{Me}-$ dian follow-up in these publications ranges from 7 to 15 months, with a median or mean time to fracture between 2 and 25 months. All studies reported an increased risk of fracture in patients with lytic metastases. Three studies reported an association between VCF progression or de-
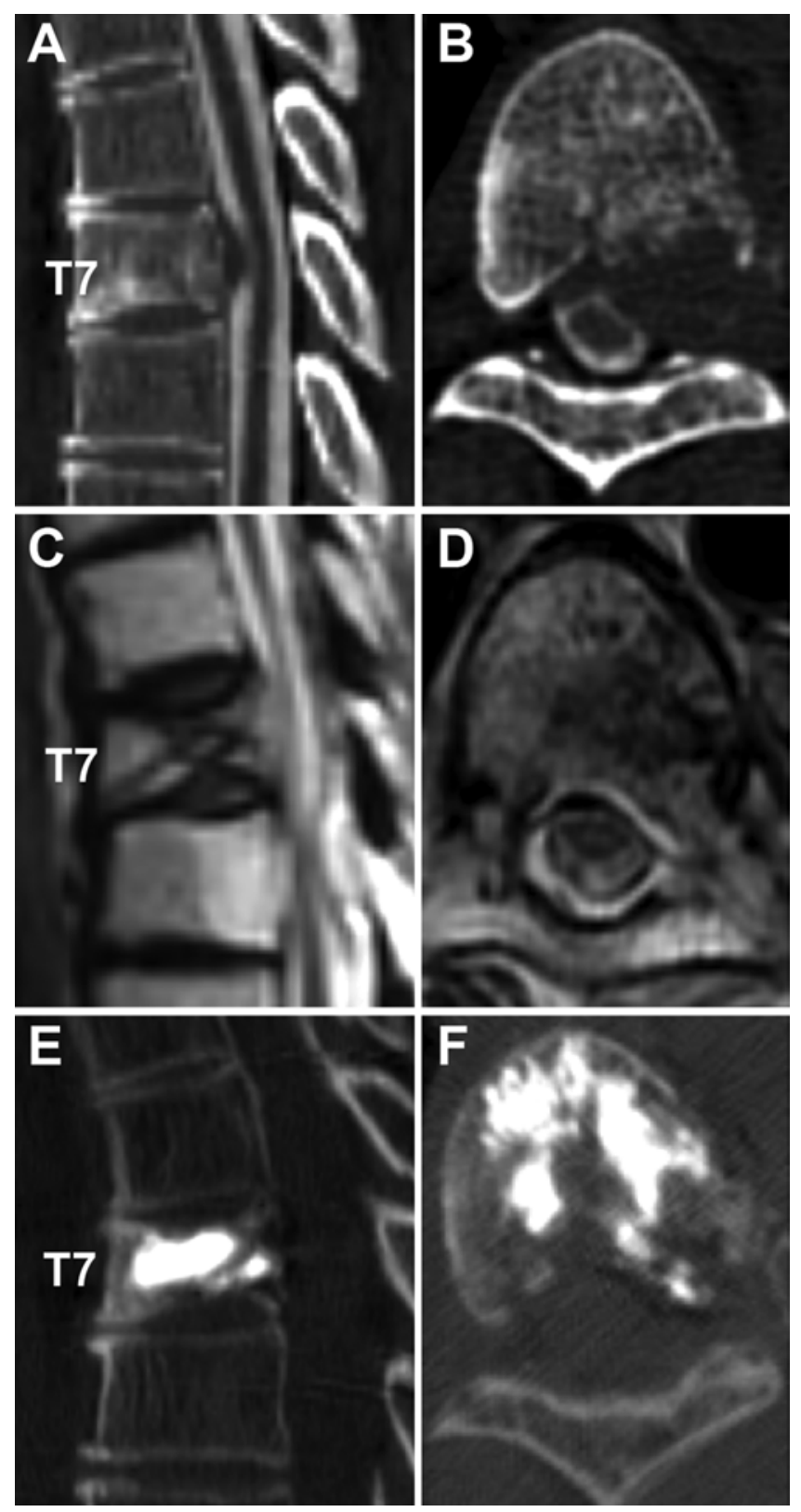

FIG. 2. Breast cancer metastasis. Sagittal (A) and axial (B) cuts from CT myelograms obtained for SRS planning, showing epidural extension without cord compression. Sagittal (C) and axial (D) cuts from T2weighted MR images illustrating T-7 VCF 13 months post-SRS. Sagittal (E) and axial (F) cuts from intraprocedural CT images obtained during kyphoplasty.

velopment in patients with preexisting VCF and deformity. ${ }^{3,5,17}$ Individual studies have also cited increasing dose per fraction, age $>55$ years, increasing vertebral body tumor involvement, thoracolumbar junction and lumbar level metastases, tumor progression, lung and/or hepatocellular histology, solitary metastases, and lack of MRI for treatment planning as risk factors for VCF. ${ }^{3,5,8,15,17,20}$ Variability in patient selection, fracture detection, presence of tumor, follow-up, and radiation methods may account for the wide range in the reported radiographic fracture risk and time to fracture. 

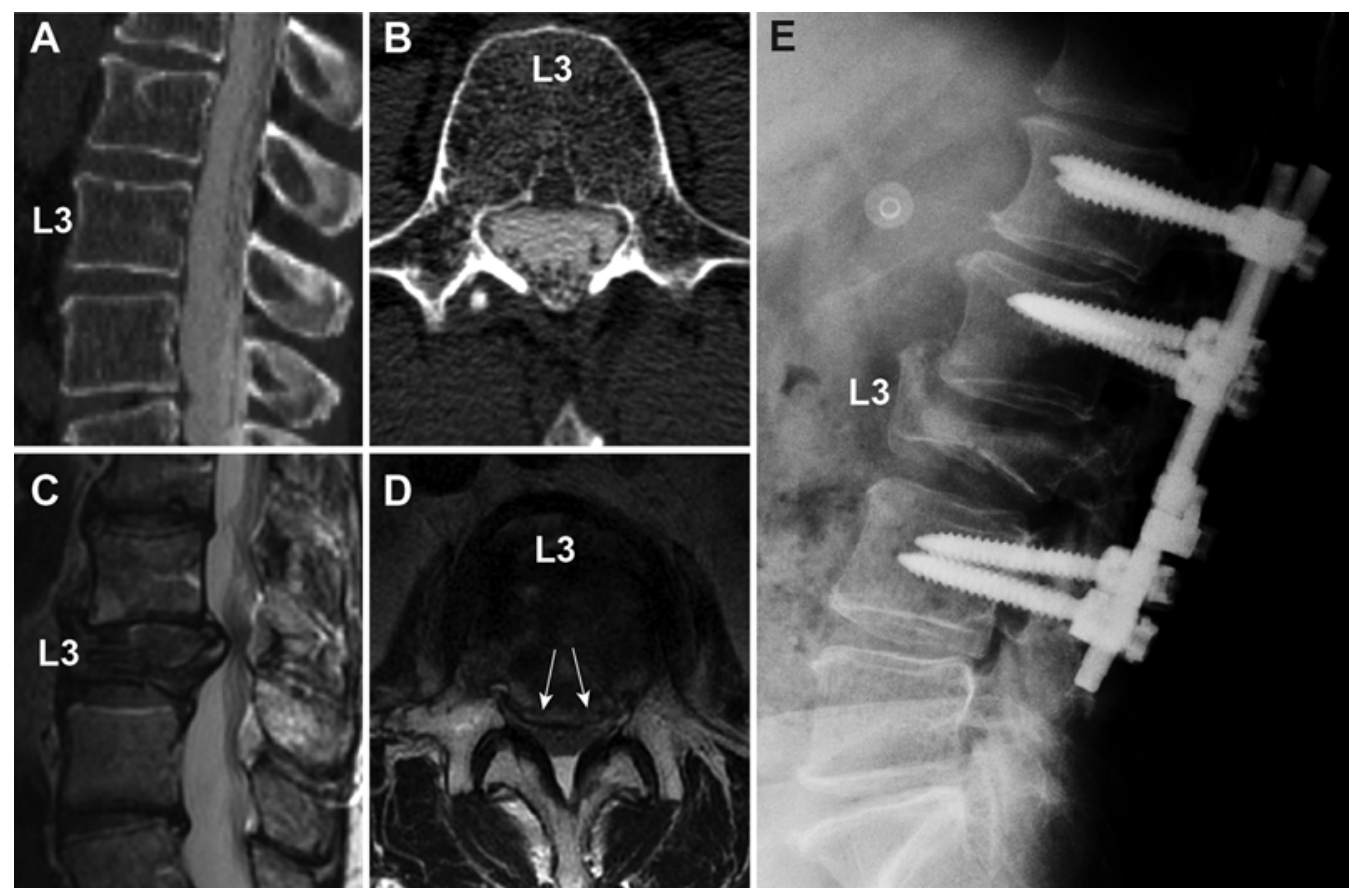

FIG. 3. Prostate cancer metastasis. Sagittal (A) and axial (B) cuts from CT myelograms obtained for SRS planning. Sagittal (C) and axial (D) cuts from T2-weighted MR images illustrating L-3 VCF with canal compromise 28 months post-SRS (arrows indicate epidural extension). Postoperative radiograph (E) obtained following L-3 decompression with L1-4 fusion and instrumentation.

A paucity of data relates to the symptomatology of post-SRS VCF, with most of the above-mentioned studies reporting radiographic VCF progression rather than VCF-associated symptomatology. Symptomatic VCF can result in pain and increased narcotic utilization and can adversely affect patient quality of life and performance status. Rose et al. noted that $70 \%$ of patients with VCF progression use opioid medications, as compared with $41 \%$ of patients without VCF progression. ${ }^{15}$ Boehling et al. documented very similar narcotic utilization $(73 \%$ vs $44 \%$, respectively). ${ }^{3}$ They also reported prospectively collected pain and Karnofsky Performance Scale (KPS)

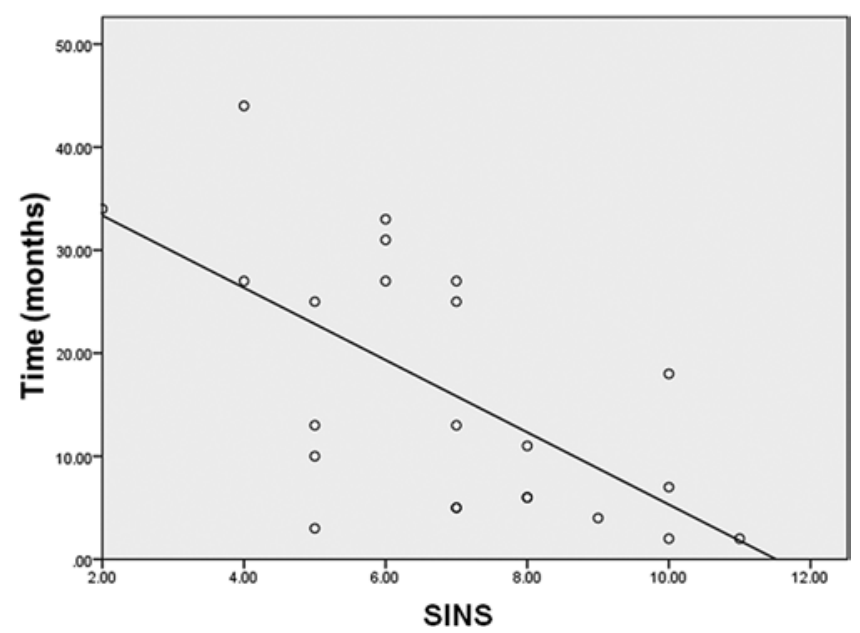

FIG. 4. Plot of SINSs at the time of SRS versus the time to VCF demonstrating a linear correlation, $R^{2}=0.4(p<0.001)$. All 19 patients who experienced VCF following upfront SRS are included. scores. Patients with post-SRS VCF had a median pain score of 3 and KPS score of 80 at the time of fracture diagnosis, whereas fracture-free patients had a median pain score of 3 and KPS score of 90. At the time of fracture, the median change in pain and KPS scores was 0 , indicating that fracture progression did not alter pain or KPS scores in at least $50 \%$ of patients. These data suggest that while more patients with post-SRS VCF may use narcotics than the fracture-free patients, radiographic post-SRS VCF does not always result in increased pain scores or a decline in performance status.

Clearly, some post-SRS VCFs result in pain, adversely affect patient quality of life and performance status, and require treatment. Patients with symptomatic tumor-associated VCF generally require stabilization, and this procedure has been shown to provide rapid and effective pain relief in this population. ${ }^{2}$ Fracture morphology determines the method of stabilization. Percutaneous cement augmentation safely and effectively treats the majority of tumorassociated VCF, with surgery reserved for more extensive fractures. ${ }^{10,11}$ Fractures that extend into posterior elements or that result in severe deformity generally require instrumented stabilization that can be performed using a minimally invasive percutaneous technique. Furthermore, focal decompression can also be readily performed using a minimally invasive technique. At our institution, open surgery is generally reserved for severe fractures requiring extensive decompression of the neural elements.

Rose et al. performed the only analysis that specifically focused on high-dose single-fraction-associated fractures; however, their analysis may be limited by a small number of patients. ${ }^{15}$ Subsequently published studies have reported a fracture risk after a wide range of frac- 
TABLE 5. Summary of post-SRS VCF studies

\begin{tabular}{|c|c|c|c|c|c|c|}
\hline Authors \& Year & No. of Patients & Levels Treated & Radiographic Fracture & Follow-Up (mos) & Time to Fracture (mos) & Stabilization \\
\hline Rose et al., 2009 & 62 & 71 & $39 \%$ & 13 & 25 & $5 \%$ \\
\hline Boehling et al., 2012 & 93 & 123 & $20 \%$ & 15 & 14 & $16 \%$ \\
\hline Cunha et al., 2012 & 90 & 167 & $11 \%$ & 7 & 2 & $10 \%$ \\
\hline Sahgal et al., 2013 & 252 & 410 & $14 \%$ & 12 & 2.5 & $10 \%$ \\
\hline Sung \& Chang, 2014 & 72 & 72 & $36 \%$ & 11 & 2 & $21 \%$ \\
\hline Jawad et al., 2016 & 580 & 594 & $6 \%$ & 10 & 3 & $4 \%$ \\
\hline Present study & 323 & 551 & NA & 12 & 13 & $8 \%$ \\
\hline
\end{tabular}

$\mathrm{NA}=$ not applicable.

tionation and dosing paradigms, with 2 studies raising the concern that high-dose-per-fraction radiation results in an increased risk of radiographic fractures. ${ }^{5,17}$ The abovementioned studies have reported that $5 \%-21 \%$ of patients required stabilization of post-SRS VCFs, with the majority of patients treated with percutaneous cement stabilization.

In the current study we provided follow-up on the Rose et al. findings and analyzed the largest cohort of patients treated with 24-Gy single-fraction SRS, with a specific focus on clinically symptomatic VCF requiring stabilization. The $8 \%$ rate of patients treated with 24-Gy single-fraction SRS who required stabilization compares favorably with the rates reported after treatment with lower-dose-perfraction radiation (Table 5). Unfortunately, since the studies with comparable rates did not specifically report how many patients with VCF were symptomatic and therefore required surgery, we cannot determine how many of the patients who underwent high-dose-per-fraction radiation actually required stabilization.

A subanalysis of patients with post-SRS VCF revealed that fractures occurred earlier in those with higher SINSs. Prior studies have found an association between the risk of radiographic fracture and SINS as well as some of the individual SINS components. ${ }^{3,5,15,17,20}$ More recently, a prospective multiinstitutional study demonstrated a predictive value of the SINS at the time of SRS for developing a VCF, with higher scores predisposing patients to symptomatic versus radiographic fractures. ${ }^{9}$ Our data confirm the utility of the SINS in patients undergoing spinal SRS and provide evidence of a direct relationship between higher SINSs and earlier symptomatic fractures (Fig. 4). An SINS analysis of the entire patient cohort was not performed in our study since the relationship between the SINS and fracture risk has been convincingly shown by earlier publications.

In our study, the largest one to focus on single-fraction SRS to date, our population differs from those in previous studies by its inclusion of patients who underwent kyphoplasty or instrumented stabilization of the irradiated levels. Our experience indicated that post-SRS fractures occur even after a vertebral level has been previously stabilized with cement or instrumentation; therefore, patients from such cases were included. Patients can experience VCFs after separation surgery as posterolateral stabilization is utilized without aggressive reconstitution of the anterior column. We have observed isolated cases of post-SRS fractures in the middle of spinal constructs and resultant rod fractures; however, the rate of symptomatic hardware failure is low. Percutaneous cement stabilization provides salvage stabilization in some instances of intraconstruct fractures without requiring invasive restabilization operations..$^{21}$ Postkyphoplasty fractures can be avoided by optimizing the cement fill technique, and investigators are on the way to determining if complete top-to-bottom fill of the vertebral body can prevent refracture.

Adjacent-level fractures post-SRS have not been previously described. Conventional 2-mm expansion of the planning target volume contour around the clinical target volume often leads to the inclusion of adjacent-level endplates into the planning target volume, resulting in the delivery of full prescription radiation doses to adjacent endplates. ${ }^{4}$ Such vertical expansion may not be required for tumor control since the intervertebral disc generally provides an excellent barrier to tumor spread..$^{18}$ However, current image-guided radiotherapy techniques generally provide superior spatial precision in radiation delivery in the axial plane as compared with the coronal and sagittal planes. Therefore, adjacent-level endplates may receive high doses of radiation even if they are excluded from treatment contours. These data serve as preliminary evidence that adjacent endplates may need to be considered organs at risk, and additional studies may be required to determine the frequency of adjacent-level fractures.

In the present study, all adjacent-level fractures occurred in patients with fractures at the treated levels. Such a correlation may indicate that these patients are predisposed to fractures because of severe osteoporosis, impaired bone remodeling, or particular radiosensitivity. Adjacent-level fractures generally occurred simultaneously with the fractures at the treated level. Additional biomechanical stresses of the levels adjacent to diseased levels may also require consideration and provide an alternative or complementary explanation for adjacent-level fractures in patients with VCF.

In cancer patients, bone health can be adversely affected by the local or systemic effect of the tumor, as well as the anticancer therapy. ${ }^{14}$ Radiation has been shown to harm bone through numerous localized and systemic effects, often leading to ablation of the vascular supply, decreased osteoblast and increased osteoclast activity, replacement of biologically active red marrow with inactive yellow marrow, chondrocyte loss, ${ }^{13}$ and direct destruction of collagen and inorganic matrix. ${ }^{7}$ While the majority of radiation-associated osseous toxicity mechanisms result in delayed toxicity, acute toxicity due to direct injury to the 
bone matrix is possible. Bone necrosis has been described in numerous skeletal structures, occurring at widely disparate time points. While the literature lacks clear consensus about the association between radiation dose and adverse skeletal events, several studies have reported a higher risk of jaw osteonecrosis with increasing radiation doses..$^{12,19}$ As experience with single-fraction SRS is gained, a more sophisticated understanding of the procedure's side effects and their underlying causes is sought. Al-Omair et al. reported 2 biopsy-confirmed cases of post-SRS osteonecrosis in the spine and found devitalized bone with necrotic debris mixed with microscopic adenocarcinoma fragments 2 years after post-SRS in the first case and bone marrow replacement by fibrosis and significantly thinned trabeculae 3 years post-SRS in the second. ${ }^{1}$ Both of these cases feature delayed radiation toxicity. Studies such as the Radiation Therapy Oncology Group trial 0631 are generating prospective multiinstitutional data directly comparing external beam radiation therapy to SRS and are likely to distinguish the differences between these 2 methods with respect to local tumor control and side effects. ${ }^{16}$

In the current study we report that $8 \%$ of patients developed VCF requiring stabilization after undergoing 24-Gy single-fraction SRS. This rate compares favorably with the literature's documented risk of symptomatic fractures after spinal SRS delivered at various fractionations. The heterogeneous patient population in and the retrospective nature of our study limit the depth of the analysis that could be effectively performed to account for systemic risk factors associated with fractures and the symptomatology of fractures that did not require stabilization. Furthermore, the small number of patients who developed fractures requiring stabilization and the heterogeneity of the tumor and vertebral body morphology among these patients further limit our analysis of risk factors. Prospective multiinstitutional studies with attention to post-SRS toxicity are needed to further characterize the patient population at risk for symptomatic fractures.

\section{Conclusions}

Symptomatic VCF requiring stabilization developed in $7.2 \%$ of patients within 5 years after 24-Gy single-fraction SRS for the treatment of spinal metastases. Currently, the majority of such fractures can be treated with percutaneous instrumented stabilization or cement augmentation. Some patients experienced adjacent-level fractures, which has not been previously reported. Higher SINSs were associated with earlier VCF stabilization.

\section{References}

1. Al-Omair A, Smith R, Kiehl TR, Lao L, Yu E, Massicotte EM, et al: Radiation-induced vertebral compression fracture following spine stereotactic radiosurgery: clinicopathological correlation. J Neurosurg Spine 18:430-435, 2013

2. Berenson J, Pflugmacher R, Jarzem P, Zonder J, Schechtman K, Tillman JB, et al: Balloon kyphoplasty versus non-surgical fracture management for treatment of painful vertebral body compression fractures in patients with cancer: a multicentre, randomised controlled trial. Lancet Oncol 12:225-235, 2011
3. Boehling NS, Grosshans DR, Allen PK, McAleer MF, Burton AW, Azeem S, et al: Vertebral compression fracture risk after stereotactic body radiotherapy for spinal metastases. J Neurosurg Spine 16:379-386, 2012

4. Cox BW, Spratt DE, Lovelock M, Bilsky MH, Lis E, Ryu S, et al: International Spine Radiosurgery Consortium consensus guidelines for target volume definition in spinal stereotactic radiosurgery. Int J Radiat Oncol Biol Phys 83:e597e605, 2012

5. Cunha MV, Al-Omair A, Atenafu EG, Masucci GL, Letourneau D, Korol R, et al: Vertebral compression fracture (VCF) after spine stereotactic body radiation therapy (SBRT): analysis of predictive factors. Int J Radiat Oncol Biol Phys 84:e343-e349, 2012

6. de Moraes FY, Taunk NK, Laufer I, Neves-Junior WFP, Hanna SA, de Andrade Carvalho H, et al: Spine radiosurgery for the local treatment of spine metastases: Intensity-modulated radiotherapy, image guidance, clinical aspects and future directions. Clinics (Sao Paulo) 71:101-109, 2016

7. Green DE, Adler BJ, Chan ME, Lennon JJ, Acerbo AS, Miller LM, et al: Altered composition of bone as triggered by irradiation facilitates the rapid erosion of the matrix by both cellular and physicochemical processes. PLoS One 8:e64952, 2013

8. Jawad MS, Fahim DK, Gerszten PC, Flickinger JC, Sahgal A, Grills IS, et al: Vertebral compression fractures after stereotactic body radiation therapy: a large, multi-institutional, multinational evaluation. J Neurosurg Spine 24:928-936, 2016

9. Lee SH, Tatsui CE, Ghia AJ, Amini B, Li J, Zavarella SM, et al: Can the spinal instability neoplastic score prior to spinal radiosurgery predict compression fractures following stereotactic spinal radiosurgery for metastatic spinal tumor?: a post hoc analysis of prospective phase II single-institution trials. J Neurooncol 126:509-517, 2016

10. Moliterno J, Veselis CA, Hershey MA, Lis E, Laufer I, Bilsky $\mathrm{MH}$ : Improvement in pain after lumbar surgery in cancer patients with mechanical radiculopathy. Spine J 14:2434-2439, 2014

11. Moussazadeh N, Rubin DG, McLaughlin L, Lis E, Bilsky MH, Laufer I: Short-segment percutaneous pedicle screw fixation with cement augmentation for tumor-induced spinal instability. Spine J 15:1609-1617, 2015

12. Nabil S, Samman N: Risk factors for osteoradionecrosis after head and neck radiation: a systematic review. Oral Surg Oral Med Oral Pathol Oral Radiol 113:54-69, 2012

13. Pacheco R, Stock H: Effects of radiation on bone. Curr Osteoporos Rep 11:299-304, 2013

14. Rizzoli R, Body JJ, DeCensi A, Reginster JY, Piscitelli P, Brandi ML: Guidance for the prevention of bone loss and fractures in postmenopausal women treated with aromatase inhibitors for breast cancer: an ESCEO position paper. Osteoporos Int 23:2567-2576, 2012 (Erratum in Osteoporos Int 23:2577, 2012)

15. Rose PS, Laufer I, Boland PJ, Hanover A, Bilsky MH, Yamada J, et al: Risk of fracture after single fraction image-guided intensity-modulated radiation therapy to spinal metastases. J Clin Oncol 27:5075-5079, 2009

16. Ryu S, Pugh SL, Gerszten PC, Yin FF, Timmerman RD, Hitchcock YJ, et al: RTOG 0631 phase 2/3 study of image guided stereotactic radiosurgery for localized (1-3) spine metastases: phase 2 results. Pract Radiat Oncol 4:76-81, 2014

17. Sahgal A, Atenafu EG, Chao S, Al-Omair A, Boehling N, Balagamwala EH, et al: Vertebral compression fracture after spine stereotactic body radiotherapy: a multi-institutional analysis with a focus on radiation dose and the spinal instability neoplastic score. J Clin Oncol 31:3426-3431, 2013

18. Sasagawa T, Kawahara N, Murakami H, Demura S, Yoshioka $\mathrm{K}$, Yamaguchi T, et al: The route of metastatic vertebral tu- 
mors extending to the adjacent vertebral body: a histological study. J Orthop Sci 16:203-211, 2011

19. Studer G, Grätz KW, Glanzmann C: Osteoradionecrosis of the mandibula in patients treated with different fractionations. Strahlenther Onkol 180:233-240, 2004

20. Sung SH, Chang UK: Evaluation of risk factors for vertebral compression fracture after stereotactic radiosurgery in spinal tumor patients. Korean J Spine 11:103-108, 2014

21. Xu R, O’Connor K, Krol G, Yamada Y, Bilsky M, Laufer I, et al: Cement salvage of instrumentation-associated vertebral fractures. AJNR Am J Neuroradiol 35:2197-2201, 2014

22. Yamada Y, Bilsky MH, Lovelock DM, Venkatraman ES, Toner S, Johnson J, et al: High-dose, single-fraction imageguided intensity-modulated radiotherapy for metastatic spinal lesions. Int J Radiat Oncol Biol Phys 71:484-490, 2008

\section{Disclosures}

Dr. Laufer is a consultant for Globus, DePuy Synthes, and SpineWave. Dr. Lis is a consultant for Medtronic. Dr. Sciubba is a consultant for DePuy Synthes, Medtronic, Globus, Orthofix, and Stryker. Dr. Yamada is a consultant for Varian Medical Systems and a medical advisory board member of the Chordoma Foundation.

\section{Author Contributions}

Conception and design: Laufer, Virk. Acquisition of data: Virk, Han, McLaughlin, Lis. Analysis and interpretation of data: Virk, Lis. Drafting the article: Laufer, Virk, Bilsky. Critically revising the article: Laufer, Sciubba, Yamada, Bilsky. Reviewed submitted version of manuscript: Laufer, McLaughlin, Sciubba, Lis, Yamada, Bilsky. Statistical analysis: Reiner. Study supervision: Laufer.

\section{Supplemental Information}

\section{Previous Presentations}

Portions of this work were submitted in digital poster form to the AANS/CNS 31st Annual Meeting of the Section on Disorders of the Spine and Peripheral Nerves held in Phoenix, Arizona, on March 4-7, 2015, and the 30th Annual Meeting of the North American Spine Society held in San Francisco, California, on October 14-17, 2015.

\section{Correspondence}

Ilya Laufer, Department of Neurosurgery, Memorial Sloan Kettering Cancer Center, 1275 York Ave., New York, NY 10065. email: lauferi@mskcc.org. 